\title{
Early blood rise in auto-antibodies to nuclear and smooth muscle antigens is predictive of prolonged survival and autoimmunity in metastatic-non-small cell lung cancer patients treated with PD-1 immune-check point blockade by nivolumab
}

\author{
ROCCO GIANNICOLA ${ }^{1}$, GRAZIELLA D'ARRIGO ${ }^{2}$, CIRINO BOTTA $^{3}$, \\ RITA AGOSTINO $^{1}$, PIETRO DEL MEDICO ${ }^{1}$, ANTONIA CONSUELO FALZEA ${ }^{1}$, VITO BARBIERI ${ }^{3}$, \\ NICOLETTA STAROPOLI ${ }^{3}$, TERESA DEL GIUDICE $^{3}$, PIERPAOLO PASTINA ${ }^{4}$, VALERIO NARDONE ${ }^{4}$, \\ MARIKA MONORITI ${ }^{5}$, GRAZIELLA CALABRESE ${ }^{6}$, GIOVANNI TRIPEPI ${ }^{2}$, LUIGI PIRTOLI ${ }^{4,7}$, \\ PIERFRANCESCO TASSONE ${ }^{3,8}$, PIEROSANDRO TAGLIAFERRI ${ }^{3,8}$ and PIERPAOLO CORREALE ${ }^{1}$ \\ ${ }^{1}$ Medical Oncology Unit, 'Bianchi-Melacrino-Morelli' Grand Metropolitan Hospital; ${ }^{2}$ Statistical Unit, National Council \\ of Research (CNR), Grand Metropolitan Hospital-IFC, I-89124 Reggio di Calabria; ${ }^{3}$ Medical Oncology Unit, \\ Department of Experimental and Clinical Medicine, Magna Graecia University, I-88100 Catanzaro; \\ ${ }^{4}$ Radiation Oncology Unit, Siena University Hospital, I-53100 Siena; ${ }^{5}$ Autoimmunity Laboratory, \\ 'Bianchi-Melacrino-Morelli' Grand Metropolitan Hospital; ${ }^{6}$ Radiology Unit, 'Bianchi-Melacrino-Morelli' \\ Grand Metropolitan Hospital, I-89124 Reggio di Calabria, Italy; ${ }^{7}$ Department of Biology, College of \\ Science and Technology, Temple University, Philadelphia, PA 19122, USA; ${ }^{8}$ Translational Oncology Unit, \\ Department of Experimental and Clinical Medicine, Magna Graecia University, I-88100 Catanzaro, Italy
}

Received November 2, 2018; Accepted April 10, 2019

DOI: $10.3892 / \mathrm{mco} .2019 .1859$

\begin{abstract}
Immune-checkpoint blockade by Nivolumab, a human monoclonal antibody to programmed cell death receptor-1, is an emerging treatment for metastatic non-small cell lung cancer (mNSCLC). In order to prolong patient survival, this treatment requires a continuous cross-priming of tumor derived-antigens to supply fresh tumor-specific immune-effectors; a phenomenon that may also trigger auto-immune-related adverse events (irAEs). The present study therefore investigated the prognostic value of multiple autoimmunity-associated parameters in patients with mNSCLC who were undergoing Nivolumab treatment. This retrospective study included $92 \mathrm{mNSCLC}$ patients who received salvage therapy with Nivolumab ( $3 \mathrm{mg} / \mathrm{kg}$, biweekly) between September 2015 and June 2018. Log-rank test, Mantel-Cox and $\mathrm{McPherson}$ analyses were conducted to correlate patient progression-free survival (PFS) and overall survival (OS) with different parameters including blood cell counts, serum
\end{abstract}

Correspondence to: Dr Pierpaolo Correale, Medical Oncology Unit, 'Bianchi-Melacrino-Morelli' Grand Metropolitan Hospital, Via Melacrino 1, I-89124 Reggio di Calabria, Italy

E-mail: correalep@yahoo.it; pierpaolo.correale@ospedalerc.it

Key words: PD-1/PDL-1-blockade, irAEs, auto-antibodies, mNSCLC inflammatory markers and auto-antibodies (AAbs). A median PFS and OS of 10 [inter-quartile range (IQR): 5.8-14.2] and 16 [IQR: 6.2-25.8] months, respectively, were recorded, which did not correlated with age, histology or the number of previous chemotherapy lines. Male gender, the type of therapeutic regimens received prior to Nivolumab, and the occurrence of irAEs were revealed to be positive predictors of prolonged survival $(\mathrm{P}<0.05)$. Early detection (within 30 days) of $>1 \mathrm{AAbs}$ among anti-nuclear antigens (ANAs), extractable nuclear antigens (ENAs) and anti-smooth cell antigens (ASMAs) correlated with prolonged PFS [hazard ratio (HR) $=0.23 ; 95 \%$ confidence interval (CI): $0.08-0.62 ; \mathrm{P}=0.004]$ and $\mathrm{OS}$ [HR=0.28 (95\% CI: 0.09-0.88), $\mathrm{P}=0.03$ ], with the type of treatment received prior to nivolumab $(\mathrm{P}=0.007)$ and with the risk of irAEs $(\mathrm{P}=0.002)$. In conclusion, increased serum levels of ANA, ENA and/or ASMA are consequential to Nivolumab administration and are predictive of a positive outcome in mNSCLC patients.

\section{Introduction}

Non-small cell lung cancer (NSCLC) represents the most common type of malignancy and the leading cause of cancer-associated mortality worldwide $(1,2)$. Platinum doublet chemotherapy, alone or in combination with radiation therapy, is the standard frontline treatment for patients with advanced disease (stages IIIB-IV), with a good performance status [Eastern Cooperative Oncology Group (ECOG) 0-1], no actionable mutations/rearrangements/translocations in epidermal growth factor receptor (EGFR; $8-10 \%$ of 
cases), anaplastic lymphoma kinase [ALK]/echinoderm microtubule-associated protein-like [EML] (3-4\%) requiring specific drugs or over-expression $(>50 \%)$ of the programmed cell death receptor ligand-1 (PDL-1; 15-20\%), which allows the use of specific programmed cell death-receptor-1 (PD-1) blockade with Pembrolizumab (3-6). Furthermore, the addition of Bevacizumab, a monoclonal antibody (mAb) to the vascular endothelial growth factor (VEGF), to the platinum doublet is also recommended in patients with non-squamous histology and a low risk of bleeding (7). Taken together, these treatments are associated to a median progression-free survival (PFS) and overall survival (OS) of 7-8 and 12-13 months, respectively (3-7). Immune-checkpoint blockade with mAbs to PD-1 (Nivolumab and Pembrolizumab) or PDL-1 (Atezolizumab) have been approved for the salvage treatment of these patients with promising results in terms of the benefits and survival, and is progressing towards successful approval as a first line treatment in selected cases (8-10).

Nivolumab, in particular, is a fully human immunoglobulin-G4 mAb to PD-1 and is presently recommended for the treatment of malignant melanoma, kidney cancer, urothelial cancer, head and neck cancer, and metastatic NSCLC (mNSCLC) (8-11). Although its use may yield a significant benefit and a prolonged survival in $\sim 20 \%$ of patients with mNSCLC, this treatment may be hampered by severe autoimmune adverse events, such as immune-related adverse events (irAEs), and high economic costs (12). At present, patients are administered PD-1/PDL-1 blockade treatment on an empirical basis, as no reliable response biomarkers that are able to guide the patients' selection have been identified, with the exception of the immune-histochemical expression of PDL-1 being $>50 \%$ for first line Pembrolizumab (15-25\% of cases) or a high tumor mutation burden for Nivolumab that occurs in $<5 \%$ of the cases and requires advanced technological approaches that cannot be utilized worldwide yet (12-14).

In order to identify reliable biomarkers for patient response to Nivolumab, the present study took into consideration the hypothesis that the PD-1/PDL-1axis is a peripheral mechanism of $\mathrm{T}$ cell inhibition and that its blockade triggers a fast cytolytic effect in the tumor tissue, throughout the rescue of immune-check point deactivated tumor infiltrating cytotoxic $\mathrm{T}$ cells (CTLs). The presence of these immune-effectors represents an immunological response to the tumor associated antigens (TAAs) and tumor specific antigens (TSAs) produced by transformed cells as a natural consequence of their carcinogenetic process, which pre-exists the use of the immune-checkpoint blocker mAbs $(15,16)$. In this context, the PD-1/PDL-1 blockade and the consequent CTL rescue give rise to a fast $\mathrm{T}$ cell-mediated cytolytic response in tumor sites that will be rapidly terminated if there is not a continuous self-sustained and prolonged supply of new active tumor-specific immune-effectors from central lymphoid organs (17). Experimental evidence has suggested that the latter process defined as immune-priming, may be critical for patient survival as it is required to avoid the exhaustion of the most specific and active CTL clones in the tumor, and to prevent an adaptive response by the tumor targets (17). On the other hand, the generation of new immune-effectors does not occur in the tumor, but rather in central lymphoid organs for the cross-presentation (immune-priming) of antigenic material released in the blood stream and lymphatic vessels by tumor tissues. Tumor-derived antigens are then taken up and processed by professional antigen presenting cells such as, dendritic cells (DCs) and activated B cells, and cleaved in small major histocompatibility complex (MHC) haplotype specific epitope peptides that are subsequently exposed on their membrane for $\mathrm{T}$ cell precursor recognition and activation (18).

A number of studies have shown that the efficacy of immune-effectors and antigen cross-priming may be supported by specific anticancer treatments (including radiotherapy, chemotherapy, steroid hormones, and immune-adjuvant agents), the hypoxic response and/or tumor associated inflammation (19-27). Tumor irradiation, several anticancer drugs, as well as specific anti-angiogenic treatments (metronomic chemotherapy, bevacizumab and multi-kinase inhibitors) may, in fact, induce immunogenic cell death, antigen modulation and release in the tumor tissues, and may also interfere with the activity of many different immunosuppressive cell lineages, such as myeloid derivative suppressor cells and regulatory $\mathrm{T}$ cells $\left(\mathrm{T}_{\mathrm{reg}}\right)$ (19-27). In this regard, several immunotherapy studies in mouse models and cancer patients have demonstrated that host survival upon treatment with cancer vaccines, immunologic treatments as well as radiotherapy (abscopal effect) was correlated with the ability of the experimental treatment to trigger an immunological antigen cascade and promote immune-priming to TAAs and TSAs (19-26) with amplified multi-antigen specific immune-responses. This effect has been recognized as a direct consequence of the treatment that was able to trigger the release of neo-antigens, producing powerful immunological danger signals (such as Calreticuline, Heath-shock proteins and damage-associated molecular patterns) that are able to alert the immune-surveillance system to a prompt an adequate response (19-26). The activation of an antigen cascade is therefore responsible for the appearance of multiple and more active tumor specific CTL precursors, TAA-specific antibodies and autoimmunity, and in turn, auto-antibodies (AAbs) such as those detected in patients with common autoimmune diseases (28-37). Thus the present study performed retrospective analysis to investigate the ability of several clinical, immune-biological parameters that reflect the aforementioned considerations, in order to predict the survival of patients with mNSCLC who received salvage treatment with Nivolumab.

\section{Materials and methods}

Patient sample, treatment and monitoring. The present multi-institutional retrospective analysis included a database of $120 \mathrm{mNSCLC}$ patients who were sequentially enrolled to receive salvage biweekly therapy with Nivolumab at the Medical Oncology Unit, Grand Metropolitan Hospital in Reggio Calabria, at the Radiation Oncology Unit, Siena University Hospital, and at the Medical and Translational Oncology Units, at AOU 'Mater Domini-Magna Graecia' University Hospital of Catanzaro, Italy, between September 2015 and June 2018. The present study was performed using a representative group of 92 cases $(76.7 \%)$, who had been screened for $\mathrm{AAb}$ detection and immune-biological analysis as well as treatment. Due to the retrospective nature of the 
study, the AAb assays could not be evaluated in 28 patients, as their serum was not available at baseline. These patients were consequently excluded from the statistical analysis; this did not influence the results of the statistical analysis, as it was completely casual. All of the enrolled patients received intravenous Nivolumab at a dosage of $3 \mathrm{mg} / \mathrm{kg}$ in $60 \mathrm{~min}$ on a biweekly basis. The treatment was continued until clear progression, unacceptable toxicity or mortality. These patients, prior to PD-1 blockade, had received at least 1 previous line of platinum-based doublet $+/$ - bevacizumab, presented a performance status $<2$, complete physical examination reports, histological samplings, and hematological, biochemical, immune-biological, imaging and instrumental monitoring.

Clinical history and physical examinations with recordings of adverse events were assessed every 2 weeks according to the best clinical practice. A computed tomography-scan was performed every 3 months or in any case showing clinical signs of Progressive Disease or an increase in any specific tumor marker, and evaluated according to the iRECIST Criteria (37).

Patients were monitored for blood cell counts, biochemistry, inflammatory markers [including C Reactive Protein (CRP), Erythrocyte-Sedimentation Ratio (ESR) and Lactate Dehydrogenase (LDH)] at baseline and prior to each treatment course. Detection assays for serum AAbs such as, anti-nuclear antigens (ANAs), extractable nuclear antigens (ENAs), anti-smooth cell antigens (ASMAs), anti-neutrophil cell antigen (c-ANCA and p-ANCA), anti-thyreoglobulin and anti-thyreo-peroxidases antibodies, were performed at baseline and every 4 weeks since the beginning of the treatment as described previously $(38,39)$. Patients were also monitored for FT3, FT4, thyroid stimulating hormone, adreno-corticotropic hormone (ACTH) and aldosterone blood levels on a monthly basis from the start of Nivolumab treatment.

An immuno-cytofluorometric analysis was also performed using the peripheral blood mononuclear cells of 40 patients enrolled in the Units of Siena and Catanzaro with the aim to evaluate the possible treatment-associated changes in the percentage of $\mathrm{T}_{\text {reg }}\left(\mathrm{CD}^{+} \mathrm{CD}^{+} \mathrm{CD} 25^{+} \mathrm{FoxP} 3^{+}\right)$, central memory T cells $\left(\mathrm{T}_{\mathrm{CM}} ; \mathrm{CD}^{+} \mathrm{CD}^{+} \mathrm{CD} 45 \mathrm{RA}^{-} \mathrm{CCR} 7^{+}\right)$, effector memory $\mathrm{T}$ cells $\left(\mathrm{T}_{\mathrm{EM}} ; \mathrm{CD}^{+} \mathrm{CD}^{+} \mathrm{CD}^{4} \mathrm{RA}^{-} \mathrm{CCR}^{-}\right)$, and natural killer (NK) subsets $\left(\mathrm{CD} 3{ }^{-} \mathrm{CD} 16^{+} \mathrm{CD} 56^{\mathrm{Dim}}\right)$ as described in our previous works (38-40).

Ethical consideration. All patients gave written consent for the anonymous use of their examinations for research. The retrospective analysis of the data was approved by the University Hospital of Siena Institutional Review Board. All procedures were undertaken in compliance with the ethical statements of the Helsinki Declaration (1964, amended most recently in 2008) of the World Medical Association. The Patients' Database is available on request, in accordance with Italian Law on the protection of personal data.

Statistical analysis. Data were expressed as the mean \pm standard deviation (normally distributed data), median and inter-quartile range (IQR; non-normally distributed data) or as per cent frequency (categorical data). Comparisons among groups were conducted using U-Mann Whitney test. Survival analyses were performed using Kaplan-Meier curves and univariate Cox regression analyses. The study outcomes were PFS and OS. The Cox analyses data were expressed as the hazard ratio (HR), 95\% Confidence interval (CI) and P-value. Statistical analysis was performed using SPSS for Windows software (Version 24; IBM, Corp., Armonk, NY, USA) and STATA for Windows software (Version 13; StataCorp LP, College Station, TX, USA).

\section{Results}

Patient features. The present cohort of 92 patients included 75 males and 17 females, with an average age of 66 years and a median follow up of 9 months. Squamous cell carcinoma was diagnosed in 31 cases, adenocarcinoma in 55 and undefined histology (NOS) in 6 patients. Prior to Nivolumab treatment, 51 patients had received a standard doublet chemotherapy line (either carboplatin + paclitaxel or carboplatin/cisplatin + gemcitabine or cisplatin + pemetrexed); and 41 received fractioned cisplatin $\left(30 \mathrm{mg} / \mathrm{m}^{2}\right.$ days $\left.1-3 \mathrm{q} 21\right)$ and metronomic oral etoposide (50 mg days 1-15q21) according to the mPE regimen alone (17 patients), or in combination (24 patients) with bevacizumab $(5 \mathrm{mg} / \mathrm{kg}$ day $3 \mathrm{q} 21$; mPEBev) (39-43). Overall, the latter 41 patients, treated in the Siena and Reggio-Calabria Units, received frontline treatment with a non-canonic, innovative metronomic chemotherapy, which has previously shown powerful antitumor activity and a significant immunomodulating effect (39-43).

Patients receiving the frontline $\mathrm{mPE} / \mathrm{mPEBev}$ regimen (41 patients) and those who received more canonic frontline platinum doublet (51 patients) presented similar features in terms of the following: Performance status (ECOG 0 vs. $\mathrm{ECOG}<2=\mathrm{mPE} / \mathrm{mPEBev}$ regimen: 21 vs. 20 patients; platinum doublets: 38 vs. 13 patients), sex (males vs. female $=\mathrm{mPE} / \mathrm{mPEBev}$ regimen: 32 vs. 9 patients; platinum doublets: 43 vs. 8 patients) and histology (Squamous vs. adenocarcinoma $+\mathrm{NOS}=\mathrm{mPE} / \mathrm{mPEBev}$ regimen 15 vs. 26 patients; platinum doublets: 22 vs. 29 patients).

Overall, 40 patients received $>1$ treatment line and 22 additional treatments of TKI (Erlotinib) even in the absence of driving EGFR mutation. Finally, palliative radiotherapy (25-30 Gy either hypo-fractionated in 1 day or fractionated in 5 days) on symptomatic single lesions (parenchymal, lymph-nodes, soft tissue, bone or brain) was administered in 48 patients prior to nivolumab.

Predictive values of clinical parameters. The median PFS and OS were 10.0 [IQR: 5.8-14.2] and 16.0 [IQR: 6.2-25.8] months, respectively (Fig. 1A and B). As previously reported in the literature, the mortality rate was higher in females than in males [HR: 2.29 (95\%CI: 1.13-4.62), $\mathrm{P}=0.01$ ], and a similar trend was observed for PFS [HR: 1.87 (95\%CI: 0.97-3.60), $\mathrm{P}=0.05$ ] (Fig. 1C and D). These results were not correlated with smoking habits, as 14 out of 17 were smokers. Nivolumab administration was well tolerated and the occurrence of grade 1-2 irAEs was recorded in 43 out of 92 (47.8\%) patients. These mainly consisted of cutaneous rush, poly-arthritis and thyroiditis, generally occurring concomitantly following 6-8 treatment courses. A more severe autoimmune pneumonitis was recorded in 3 cases following 6-7 treatment courses, and uveitis was recorded in 2 patients following 5 and 6 treatment courses. Hypophysitis and adrenal gland damage were not 
A
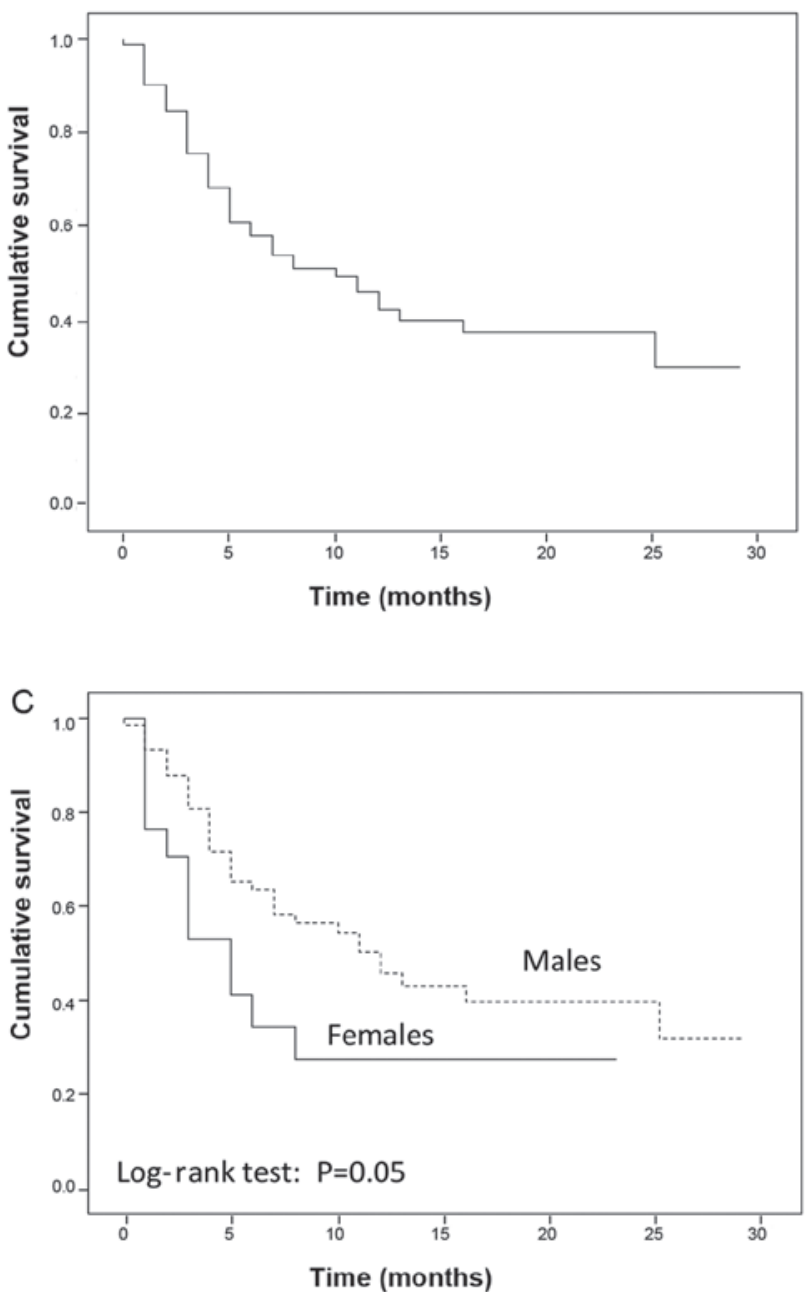

E

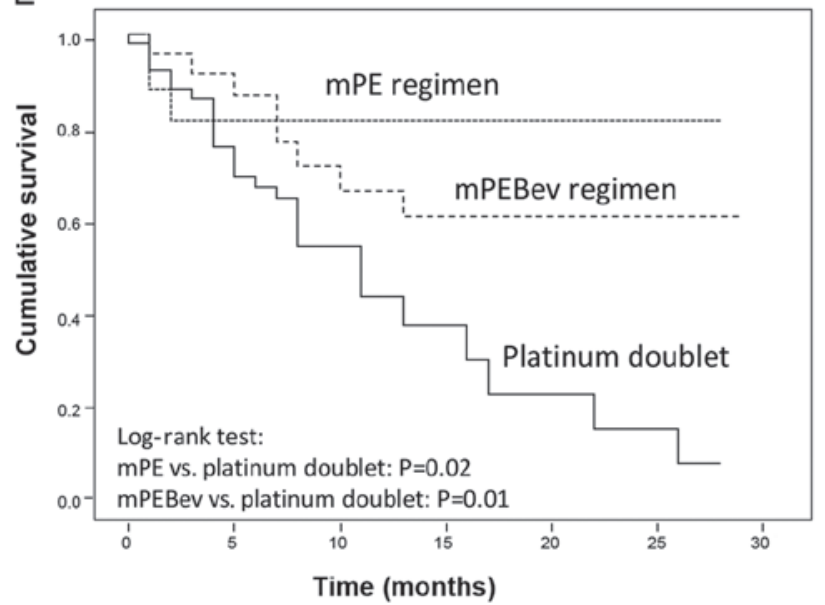

B

OS
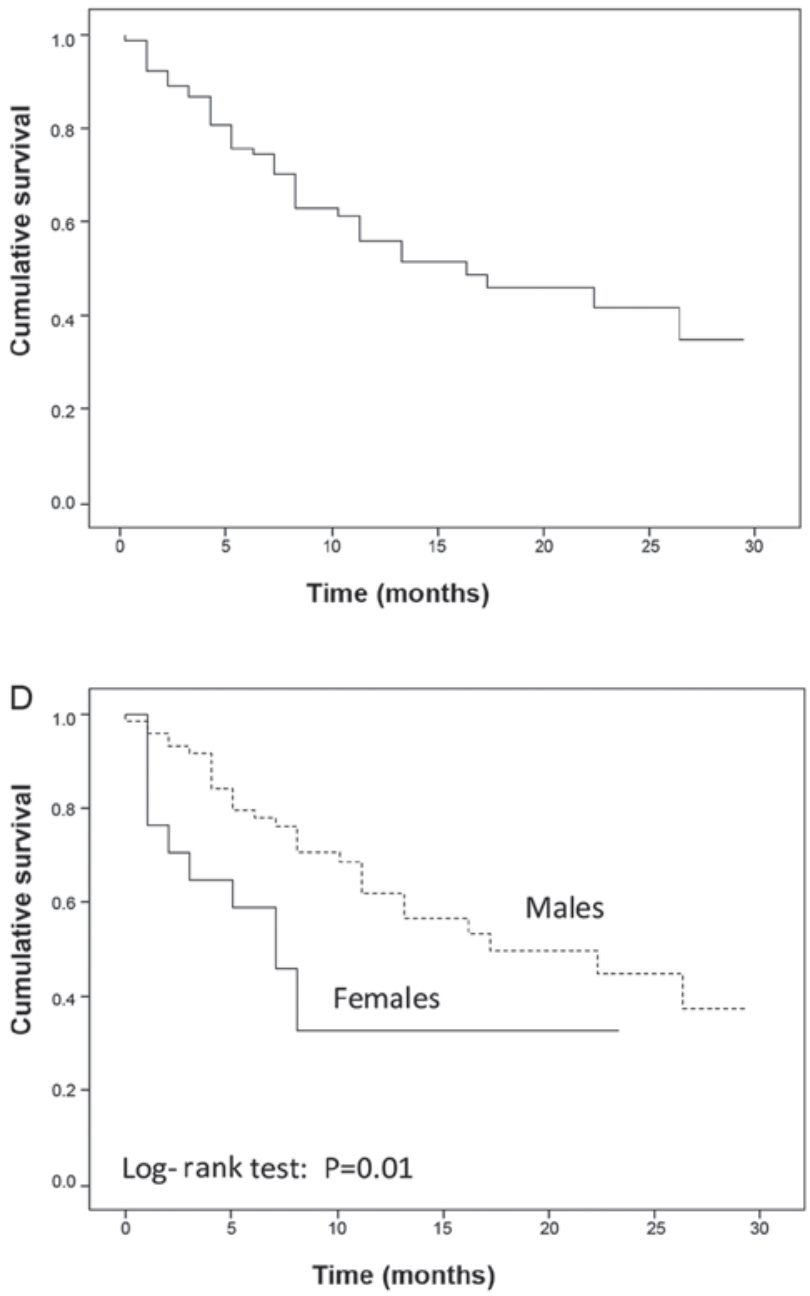

$\mathrm{F}$

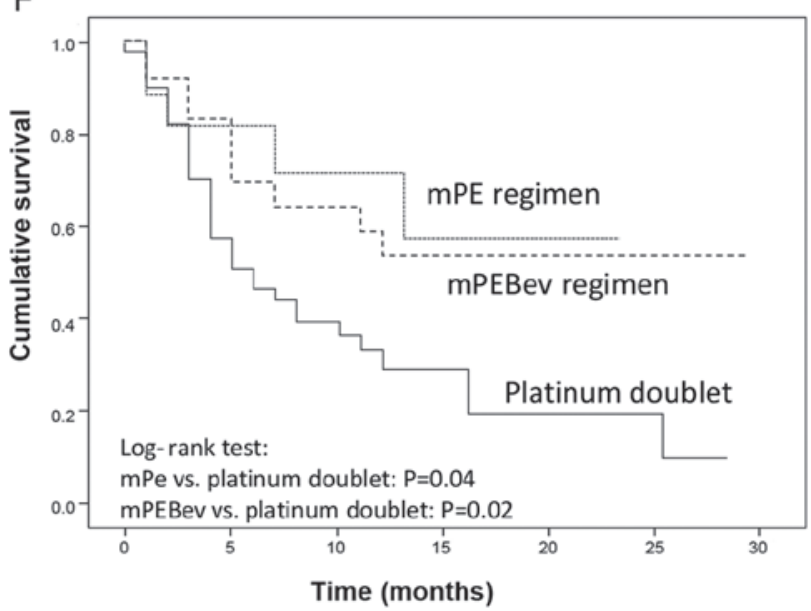

Figure 1. PFS and OS recorded in mNSCLC patients receiving Nivolumab as salvage therapy (a Log-rank test). (A and B) Results recorded on the overall population. (C and D) Results in comparison between male and female sex. (E and F) Results recorded in patients that had undergone standard chemotherapy, $\mathrm{mPE}$ regimen or mPEBev regimen prior the immune-oncological treatment with nivolumab. PFS, progression free survival; OS, overall survival; mNSCLC, metastatic non-small cell lung cancer; mPE, fractioned cisplatin (30 mg/m² days 1-3q21) and metronomic oral etoposide (50 mg days 1-15q21); mPEBEV, fractioned cisplatin (30 mg/m² days 1-3q21) and metronomic oral etoposide (50 mg days 1-15q21) in combination with bevacizumab (5 mg/kg day $3 \mathrm{q} 21)$.

demonstrated even though asymptomatic concomitant decline in $\mathrm{ACTH}$ and aldosterone serum levels were recorded in 4 patients following 6-8 treatment courses.
Treatment response and autoimmunity. The present study was unable to identify a correlation among PFS and OS, and age, smoking habitude, histology, number of previous 
A

PFS

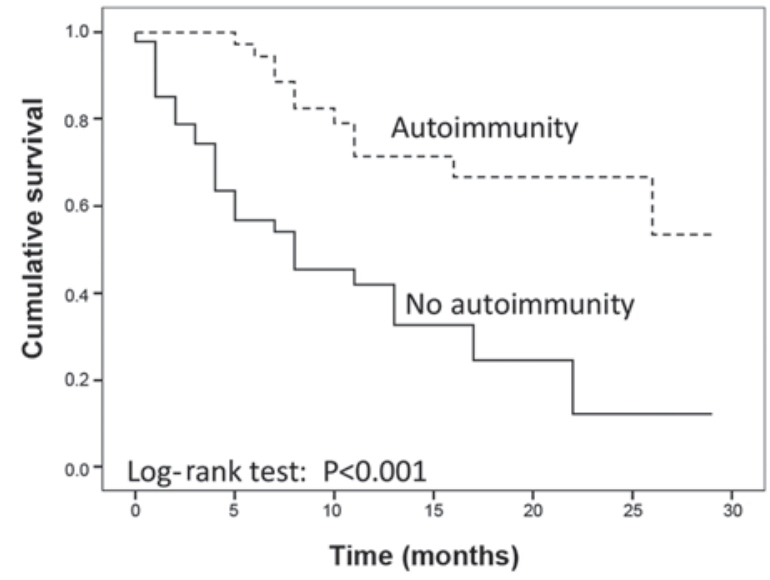

C

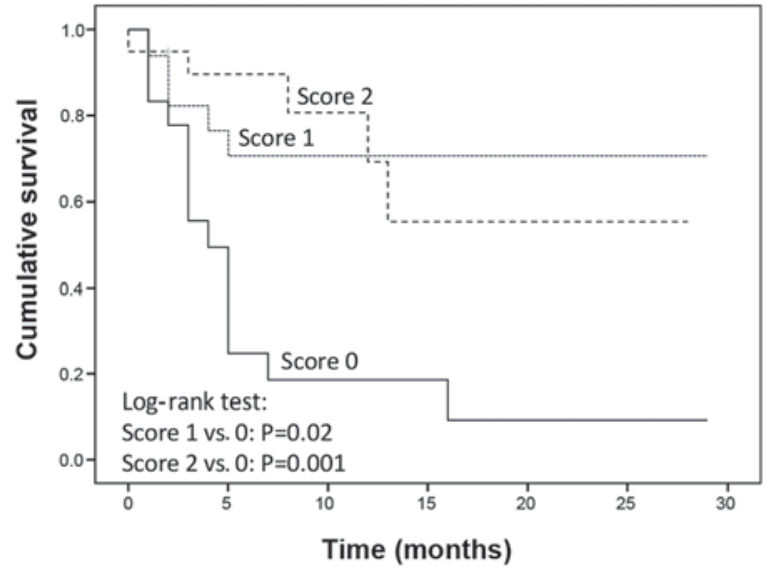

B

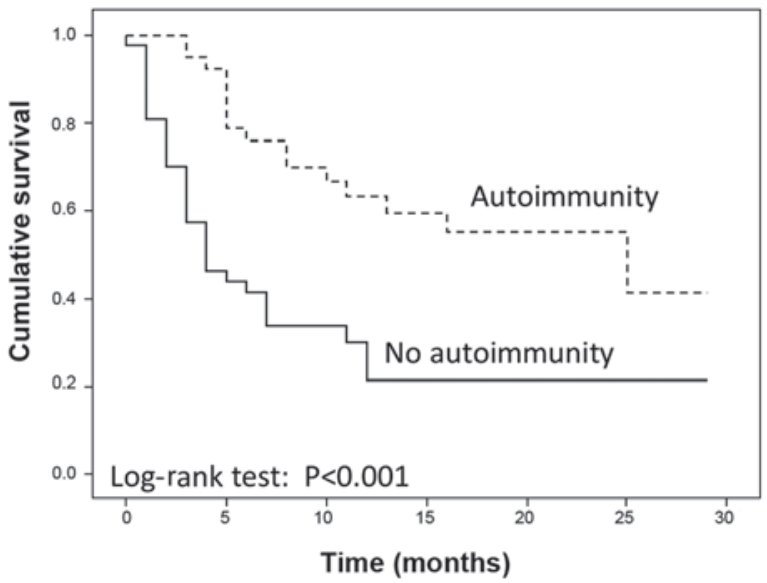

D

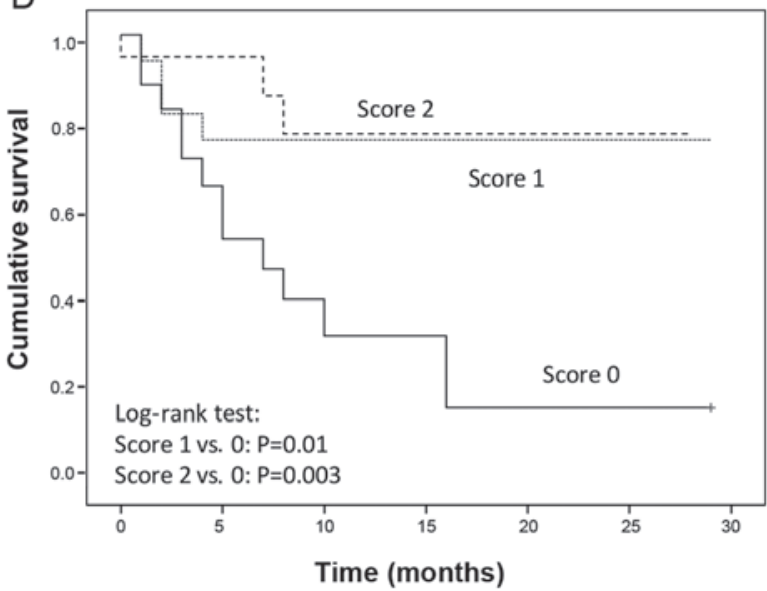

Figure 2. PFS and OS recorded in mNSCLC patients receiving Nivolumab as salvage therapy (a Log-rank test). (A and B) Comparative results between patients who showed or did not show clinical evidence of autoimmunity. (C and D) Comparative results among patients who did not exhibited an increase in serum AAbs (ANA, ENA, and ASMA), patients who exhibited an increase in one of the three AAbs (score 1) and patients who exhibited an increase in two or three AAbs (Score 2). PFS, progression free survival; OS, overall survival; mNSCLC, metastatic non-small cell lung cancer; AAbs, auto antibodies.

chemotherapy lines, TKI or radiotherapy (data not shown). On the other hand, the results revealed a significant advantage in PFS and OS in the group of patients who had received metronomic chemotherapy according to the $\mathrm{mPE}$ regimen [PFS: HR=0.40 (95\% CI: 0.16-1.03) $\mathrm{P}=0.02$; OS: HR=0.29 (95\% CI: $0.09-0.95), \mathrm{P}=0.04]$ or metronomic chemotherapy and bevacizumab [mPEBev regimen; PFS: $\mathrm{HR}=0.44(95 \%$ CI: 0.22-0.91), $\mathrm{P}=0.01$; OS: $\mathrm{HR}=0.40$ (95\% CI: 0.18-0.88), $\mathrm{P}=0.02$ ] (Fig. 1E and $\mathrm{F}$ ); compared with the group of patients who received a standard platinum doublet. A prolonged PFS and OS was finally recorded in patients who manifested irAEs [PFS: HR=0.31 (95\% CI, 0.17-0.58) P<0.001; OS: HR=0.26 (95\% CI: 0.13-0.53), $\mathrm{P}<0.001$; (Fig. 2A and B).

Immune-biological markers. Monitoring Nivolumab treatment did not reveal significant changes in the blood cell counts of neutrophils, monocytes, lymphocytes, or the neutrophil to lymphocyte ratio (NLR). Similarly, no statistically significant treatment-associated changes were detected for inflammatory markers such as CRP, ESR or LDH. In addition, the peripheral lymphocyte subsets $\mathrm{CD}^{+} \mathrm{CD}^{+}[23.1 \%(+/-9.5)$ vs. $26.7 \%$ $(+/-14.8), \mathrm{P}=0.58)], \mathrm{CD}^{+}{ }^{+} \mathrm{CD} 4^{+} \mathrm{PD}-1^{+}[5.77 \%(+/-2.72)$ vs. $8.25 \%$
(+/-6.88), $\mathrm{P}=0.169], \mathrm{T}_{\mathrm{cm}}[2 \%(+/-1.85)$ vs. 3.41\% (+/-4.03), $\mathrm{P}=0.32], \mathrm{T}_{\mathrm{em}}[4.03 \%(+/-2.39)$ vs. 5.01\% (+/-3.25), $\mathrm{P}=0.39]$, $\mathrm{CD}^{+}{ }^{+} \mathrm{CD}^{+}[50.7 \%(+/-11.09)$ vs. $42 \%(+/-14.9), \mathrm{P}=0.0163]$, $\mathrm{T}_{\text {reg }}[2.8 \%(+/-1.93)$ vs. $1.87 \%(+/-1.11) ; \mathrm{P}=0.088]$ or NKs $[7.66$ $(+/-3.62)$ vs. $7.19(+/-4.99), \mathrm{P}=0.75]$ did not show any significant treatment-associated changes following 3 treatment courses. However, a significant increase in the eosinophil cell counts was recorded, exhibiting a rapid and significant increase with treatment [baseline value vs. value after 3 treatment courses: $0.095(+/-0.013)$ vs. $0.140(+/-0.018) 10^{3}$ cells $\left./ \mathrm{mm}^{3}, \mathrm{P}=0.0014\right]$.

The present study then evaluated the patients' serumconversion of AAbs (ANA, ENA, ANCA, p-ANCA and c-ANCA), which are commonly associated with frequently occurring auto-immune diseases. Within 30 days since the beginning of the treatment 18 patients $(25.7 \%)$ became positive (Serum titration $>1 / 160$ ) for the expression of 1 of these (15 ANA and 3 ENA; scored as 1), while a further 22 (31.4\%) became contemporary positive for the expression of 2 or 3 of these (ANA, ENA and ASMA; scored as 2). Overall, there was a statistically significant increase in serum ANA (baseline vs. third treatment course: 0.37 vs. 1.13 title score; $\mathrm{P}=0.00028$ ) and ASMA (baseline vs. third treatment course: 0.076 vs. 0.41 
Table I. Cox regression analysis indicated a direct association between the risk of progression and fatality with baseline values of neutrophil and lymphocyte counts, ESR and LDH.

PFS

\begin{tabular}{cccc}
\hline HR $(95 \% \mathrm{CI})$ & P-value & HR $(95 \% \mathrm{CI})$ & P-value \\
\hline $1.13(1.01-1.26)$ & 0.04 & $1.14(1.003-1.29)$ & 0.04 \\
$1.14(0.98-1.31)$ & 0.08 & $1.19(1.02-1.40)$ & 0.03 \\
$1.03(1.01-1.04)$ & 0.002 & $1.04(1.02-1.06)$ & 0.001 \\
$1.04(1.02-1.06)$ & $<0.001$ & $1.04(1.01-1.06)$ & 0.001 \\
\hline
\end{tabular}

Variables at baseline (units)

Neutrophil cell counts $\left(10^{3} / \mathrm{mm}^{3}\right)$

Lymphocytes cell count $\left(10^{3} / \mathrm{mm}^{3}\right)$

$\operatorname{ESR}(\mathrm{mm} / \mathrm{h})$

LDH $(20 \mathrm{U} / \mathrm{l})$

PFS, progression free survival; OS, overall survival; ESR, erythrocyte sedimentation rate; LDH, lactic acid dehydrogenase; CI, confidence interval; HR, hazard ratio.

Table II. Cox regression analysis indicated a direct association between the risk of progression and fatality with changes (Delta of values at three treatment courses vs. baseline) in neutrophil counts and NLR, and an inverse correlation of the risk of both outcomes with changes in eosinophil and lymphocyte counts.

\begin{tabular}{lcccc}
\hline & \multicolumn{2}{c}{ PFS } & & OS \\
\cline { 2 - 3 } Variables at the third treatment course (units) & HR $(95 \% \mathrm{CI})$ & P-value & HR (95\% CI) & P-value \\
\hline Neutrophil cell counts $\left(10^{3} / \mathrm{mm}^{3}\right)$ & $1.13(1.03-1.24)$ & 0.009 & $1.16(1.04-1.29)$ \\
Lymphocytes cell count $\left(10^{3} / \mathrm{mm}^{3}\right)$ & $0.79(0.63-1.00)$ & 0.05 & $0.71(0.55-0.91)$ \\
Eosinophil cell counts $\left(10^{3} / \mathrm{mm}^{3}\right)$ & $0.008(0.00-0.23)$ & 0.005 & $0.03(0.001-0.771)$ & 0.04 \\
NLR & $1.19(1.06-1.34)$ & 0.005 & $1.27(1.10-1.46)$ & 0.001 \\
\hline
\end{tabular}

Change in ESR and LDH were unrelated to the outcomes. PFS, progression free survival; OS, overall survival; NLR, neutrophil lymphocyte ratio; ESR, erythrocyte sedimentation rate; $\mathrm{LDH}$, lactic acid dehydrogenase; CI, confidence interval; HR, hazard ratio.

title score; $\mathrm{P}=0.0094)$. Only 3 patients exhibited a significant increase in c-ANCA and p-ANCA levels following 12 months of treatment, and were not clustered with ANA, ENA and ASMA nor were they associated with clinical evidence of more severe irAEs (grade 3).

Statistical correlation. The present analysis demonstrated that either PFS or OS were directly correlated with the early (within 30 days) serum-conversion for one (score 1) or more (score 2) AAbs among ANA, ENA, ASMA [PFS: score 1 vs. score 0: $\mathrm{HR}=0.23$ (95\% CI: $0.08-0.65), \mathrm{P}=0.006$; score 2 vs. score 0: $\mathrm{HR}=0.23$ (95\% CI: 0.08-0.62), $\mathrm{P}=0.004$; OS: score 1 vs. score 0: $\mathrm{HR}=0.28$ (95\% CI: $0.09-0.88), \mathrm{P}=0.03$; score 2 vs. score 0 : HR=0.19 (95\% CI: 0.05-0.68), P=0.01]; (Fig. 2C and D).

Cox regression analysis revealed a direct association between the risk of progression and death with the baseline values of neutrophil and lymphocyte cell counts, ESR and LDH (Table I). Both the risk of progression and death were correlated with treatment-associated changes (Delta value) in neutrophil counts and NLR (where the Delta value=value detected following 3 treatment courses-the value detected at baseline). Conversely, PFS and OS were not correlated with changes in CRP, ESR and LDH, or changes in the aforementioned lymphocyte subsets (data not shown). Taken together, these results suggest that a chronic inflammation status may negatively affect the treatment response to Nivolumab.
Conversely, the present analysis also revealed an inverse correlation between the risk of progression and death and treatment-associated changes (Delta value) with eosinophil and lymphocyte cell count changes (Table II).

Comparative analyses. Patients who exhibited irAEs, when compared with the other patients, had lower baseline levels of monocyte cell counts $\left[0.5710^{3} / \mathrm{mm}^{3}\right.$ (IQR $0.41-0.68$ vs. $0.7310^{3} / \mathrm{mm}^{3}$ (IQR 0.57-0.87), $\left.\mathrm{P}=0.006\right], \mathrm{CRP}$ [3.07 $\mathrm{mg} / \mathrm{dl}$ (IQR 1.64-10.44 vs. $11.49 \mathrm{mg} / \mathrm{dl}(\mathrm{IQR} 3.15-36.25), \mathrm{P}=0.007]$ and LDH [376 U/1 (IQR 232-431 vs. 472 U/1 (IQR 288-704), $\mathrm{P}=0.024]$. Patients who exhibited irAEs also had a significant treatment-associated increase in the number of lymphocytes $\left[\Delta: 0.1910^{3} / \mathrm{mm}^{3}\right.$ (IQR -0.14-0.50) vs. $-0.1210^{3} / \mathrm{mm}^{3}$ (IQR -0.42-0.16), $\mathrm{P}=0.037]$ and monocytes $\left[\Delta: 0.0710^{3} / \mathrm{mm}^{3}\right.$ (IQR -0.10 - 0.19) vs. $-0.1110^{3} / \mathrm{mm}^{3}$ (IQR -0.29-0.08), $\mathrm{P}=0.031$ ] in comparison with the other patients. By contrast, higher baseline NLRs were correlated with a lower frequency of irAEs $[\Delta$ : -0.36 (IQR -1.59-0.68) vs. 0.20 (IQR -0.86-2.32), $\mathrm{P}=0.009]$, and a rise in AAbs.

A Chi square test finally revealed that the frequency of irAEs was correlated with both AAbs' serum conversion $(\mathrm{P}=0.002)$ and the type of therapy received prior to Nivolumab administration. Finally, the present analysis also revealed a greater frequency of irAEs in patients who had received metronomic +/- bevacizumab when compared with those who 
had received standard chemotherapy doublet prior nivolumab [69.04\% vs. $34.6 \% ; \mathrm{P}=0.007]$.

\section{Discussion}

The recent development of the PD-1/PDL-1 blockade with the newest $\mathrm{mAbs}$ represents an extraordinary challenge in term of costs, adverse events and patient monitoring. In this context, the identification of biomarkers that can predict the response of the patients' treatment selection appears to be of critical value and is urgently required. In the present retrospective study, we evaluated multiple clinical and immune-biological markers, that were directly or indirectly correlated with both immune-priming and effector phases of immune-response. The results revealed that the male gender, the occurrence of irAEs, as well as the use of the immune-modulating chemotherapy regimen, known as $\mathrm{mPE}+/$ - bevacizumab, prior to nivolumab administration, were strongly predictive of a positive outcome in terms of both PFS and OS. In addition, patients who had received the $\mathrm{mPE}$ regimen + -- bevacizumab prior to Nivolumab presented a greater risk of irAEs with the immuno-oncological treatment. These results were not surprising, considering that the $\mathrm{mPE} / \mathrm{mPEBev}$ regimen had been designed on a translational basis (BEVA2007 trial), in order to achieve a higher dose intensity of both cisplatin and etoposide to induce anti-angiogenetic effects and to the trigger immune-modulating effects associated with VEGF and angiopoietin-2 decline (21,39-43). At the end of the treatment, the BEVA2007 phase I-II trials, which enrolled a cohort of 112 mNSCLC patients [39 of whom received the $\mathrm{mPE}$ regimen and 73 were administered $\mathrm{mPE}$ in combination with bevacizumab (mPEBev)] reported a median PFS and OS of 7 (95\% CI: 6.17-7.82) and 15 (95\% CI: 13.23-16.77) months, respectively (39-43).

The immune-biological monitoring of these patients demonstrated a treatment-associated induction of the cytotoxic $\mathrm{T}_{\mathrm{H} 1}$ cytokine phenotype and with a treatment-associated

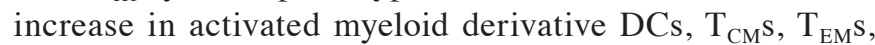
activated $\mathrm{CTLs}\left(\mathrm{CD}^{+} \mathrm{CD}^{+} \mathrm{CD}^{+} 2 \mathrm{~L}^{+}\right.$andCD $\left.3{ }^{+} \mathrm{CD}^{+} \mathrm{CD} 27^{+}\right)$, and tumor specific $\mathrm{T}$ cell precursors' frequency (39-43).

Further ancillary analysis of the BEVA2007 trial revealed that the patients who had received palliative radiotherapy in the course of the $\mathrm{mPE} / \mathrm{mPEBev}$ treatment, presented the longest survival and that this event was strictly correlated with the treatment-associated rise in activated $\mathrm{DC}$ and $\mathrm{T}_{\mathrm{cm}}(39,44)$. The results of this study supported the hypothesis of the immune-mediated effect of radiotherapy, which was able to trigger efficient immune-priming coupled with the production of immune-danger signals, becoming a powerful source of new available antigens for an efficient immune-response, which was subsequently supported by the immune-biological effects of the cytotoxic regimen $(19,32)$.

In line with the literature, the present study reported that the PD-1/PDL-1 immune-checkpoint blockade, similarly to other anti-cancer immunological treatments, was associated with the occurrence of more or less severe irAEs and that this event in turn, was predictive of prolonged survival (29-31,45-47). This phenomenon, consequential to the occurrence of an immune-primed antigen cascade and antigen migration, has been recorded in other immunotherapy-(Gvax, Ipilimumab +/- gp100, TSPP vaccine) $(48,49)$ and chemo-immunotherapy models (mPEBev, GOLFIG1 and 2 trials) $(31,39,40,50-52)$ and represents the basic concept of the radiotherapy-induced abscopal effects and is strongly predictive of positive outcome (33). The occurrence of irAEs (such as thyroiditis, polyarthritis and hypophysitis) in patients treated with Nivolumab is an unpredictable event in terms of sites and timing. It may be very difficult to identify at its early stage, and is often complicated by confounding co-morbidities and symptoms. Thus, it cannot be considered as a predictive biomarker of response; however, in an attempt to target autoimmunity as a potential biomarker of response, the present study evaluated the serum of these patients, the levels of multiple AAbs currently associated with the most common systemic auto-immune-diseases (ENA, ANA and ASMA) and vasculitis (c-ANCA and p-ANCA), and monitored the trend of inflammatory markers (CRP, ESR and $\mathrm{LDH})$; the rise in these markers has been detected in previous immunotherapy studies performed by our group (31,39,49-53). An early (15-30 days) serum-conversion of at least 1 of the 3AAbs among ENA, ANA and ASMA, was recorded in 57\% of the patients, an event that was highly predictive of a better outcome and occurrence of subsequent autoimmunity. It was also demonstrated that the rise in AAbs was clustered with previous $\mathrm{mPE} / \mathrm{mPEBev}$ treatment, a lower NLR value at baseline and a low inflammatory level at the baseline, while it was directly correlated with treatment-associated increases in eosinophil and lymphocyte counts. While the present study was unable to demonstrate a significant correlation between eosinophil cell count increase with both a higher frequency of irAEs or rise in AAbs, a significant inverse correlation was detected among increased NLR, irAEs and increased AAbs. The latter of which, in particular, was in line with the results of Bagley et al (54) who revealed that a baseline NLR $\geq 5$ was strongly predictive of poor outcomes in term of PFS and OS in patients with NSCLC under treatment with PD-1 inhibitors.

The fast occurrence of AAbs in these patients upon Nivolumab treatment supports the hypothesis that nivolumab-reactivated CTLs may also trigger both the immune-priming of new antigens (antigen migration) and a clear antigen cascade process resulting in the occurrence of AAbs including ANA, ENA, and ASMA. The immune-mediated damage of the tumor tissue, in fact may give rise to the immune-priming of sequestered material recognized as non-self that in turn gives rise to a humoral, as well as a cell mediated response. This phenomenon explains the rapid occurrence of Abs to nuclear antigens (ANA and ENA), smooth cells (ASMA) and the thyroid (microsomal antigens), which in the long term have provided clinical evidence of autoimmunity and are indirect signs of an efficient immune-reaction. Similar results have also been achieved in other immunotherapy trials that aimed to test Gvax in gastro-enteric malignancies; ipilimumab +/- gp100 in malignant melanoma, and the TSPP vaccine in colorectal cancer, whose administration was associated to a treatment-associated serum-conversion for anti-thyroid AAbs, NY-ESO-1 Abs, and anti-neutrophil AAbs (c/p-ANCA) respectively, which was in turn predictive of treatment response and longer survival $(39,48,49,52,53)$.

To date, no clear biomarker has been able to select patients who may benefit from treatment with Nivolumab in NSCLC. PDL-1 expression in the tumor sites is not reliable for several 
reasons including the dynamic expression on tumor-associated inflammatory cells and the presence of other PD-1 ligands (54). Similarly, a predictive value has been identified in DNA mismatch repair deficiency (MSI-high status) and in a high tumor mutation burden (TMB), which is suggestive of a greater number of potential neo-antigens and eventually, an expanded multi-antigenic CTL response to the tumor. TMB in particular, has been associated with a favorable response to Nivolumab in NSCLC patients receiving this treatment as frontline therapy. Nevertheless, next generation sequencing, which allows for TMB analysis, cannot be considered as a common practice $(14,55)$.

At the present, research on biomarkers has also focused on the expression of MHC molecules on tumor cells and the role of multiple immunosuppressive tumor infiltrating cell lineages (such as macrophages, $\mathrm{T}_{\mathrm{reg}} \mathrm{s}$, MDSCs and $\mathrm{IDO}^{+} \mathrm{DCs}$ ) with controversial results in terms of their validation as predictive biomarkers $(56,57)$. Concomitant use of Nivolumab or Pembrolizumab with platinum doublets has also been investigated reporting a better outcome in patients who had received the chemo-immuno-oncologic treatment as a frontline therapy compared with those who received the same chemotherapy alone and PD-1/PDL-1 blockade at the sign of progression. The concomitant and or sequential use of these mAbs with specific anticancer drugs, radiotherapy to induce immunogenic cell death, as well as tumor specific active specific immunotherapy (cancer vaccines), and other immune-checkpoint inhibitors is still an argument to debate.

In conclusion, the present results indicate that the early treatment-associated rise of serum AAbs ANA, ENA and ASMA, may be a surrogate marker of autoimmunity and is strongly predictive of patient response to Nivolumab in terms of PFS and long term survival. Additionally, the present study suggested the potential mechanisms that are able to trigger an antigen cascade and enhance the cross-priming of neo-antigens, which may improve the survival of these patients, thereby offering the rationale to design perspective trials aiming to evaluate the predictive value of these AAbs to select patients that may not require the addition of other checkpoint inhibitor treatment or chemotherapy following a course of Nivolumab alone. The present results also support the additional investigation of the ability of the $\mathrm{mPE} / \mathrm{mPEBev}$ regimen to improve the therapeutic effects of the PD-1/PDL-1 blockade.

\section{Acknowledgements}

Not applicable.

\section{Funding}

No funding was received.

\section{Availability of data and materials}

The datasets used and/or analyzed during the present study are available from the corresponding author on reasonable request.

\section{Authors' contributions}

RG, GDA, CB, RA and PC made substantial contributions to conception and design. PDM, ACF, VB, NS and TDG made substantial contributions in the acquisition of data. PP, VN, MM, GC, GT, LP, PTas, PTag and PC made substantial contributions in the analysis and interpretation of data. All authors contributed in drafting the manuscript and gave final approval of the version to be published.

\section{Ethics approval and consent to participate}

All patients gave written consent for the anonymous use of their data. The retrospective analysis of the data was approved by the University Hospital of Siena Institutional Review Board. All procedures were undertaken in compliance with the ethical statements of the Helsinki Declaration (1964, amended most recently in 2008) of the World Medical Association.

\section{Patient consent for publication}

Not applicable.

\section{Competing interests}

The authors declare that they have no competing interests.

\section{References}

1. Ferlay J, Soerjomataram I, Dikshit R, Eser S, Mathers C, Rebelo M, Parkin DM, Forman D and Bray F: Cancer incidence and mortality worldwide: Sources, methods and major patterns in GLOBOCAN 2012. Int J Cancer 136: E359-E386, 2015.

2. Cetin K, Ettinger DS, Hei YJ and O'Malley CD: Survival by histologic subtype in stage IV nonsmall cell lung cancer based on data from the Surveillance, epidemiology and end results program. Clin Epidemiol 3: 139-148, 2011.

3. Pilkington G, Boland A, Brown T, Oyee J, Bagust A and Dickson R: A systematic review of the clinical effectiveness of first-line chemotherapy for adult patients with locally advanced or metastatic non-small cell lung cancer. Thorax 70: 359-367, 2015.

4. Noh JM, Kim JM, Ahn YC, Pyo H, Kim B, Oh D, Ju SG, Kim JS, Shin JS, Hong CS, et al: Effect of radiation therapy techniques on outcome in N3-positive IIIB non-small cell lung cancer treated with concurrent chemoradiotherapy. Cancer Res Treat 48: 106-114, 2016.

5. Bearz A, Berretta M and Tirelli U: Clinical effectiveness and cost-effectiveness of target therapies for adult patients with locally advanced or metastatic non-small cell lung cancer: A systematic review. Curr Cancer Drug Targets 18: 405-409, 2018.

6. Horn L, Spigel DR, Vokes EE, Holgado E, Ready N, Steins M, Poddubskaya E, Borghaei H, Felip E, Paz-Ares L, et al: Nivolumab versus docetaxel in previously treated patients with advanced non-small-cell lung cancer: Two-year outcomes from two randomized, open-label, phase III trials (CheckMate 017 and CheckMate 057). J Clin Oncol 35: 3924-3933, 2017.

7. Di Costanzo F, Mazzoni F, Micol Mela M, Antonuzzo L, Checcacci D, Saggese M and Di Costanzo F: Bevacizumab in non-small cell lung cancer. Drugs 68: 737-746, 2008.

8. Pardoll DM: The blockade of immune checkpoints in cancer immunotherapy. Nat Rev Cancer 12: 252-264, 2012.

9. Meyers DE, Bryan PM, Banerji S and Morris DG: Targeting the PD-1/PD-L1 axis for the treatment of non-small-cell lung cancer. Curr Oncol 25: e324-e334, 2018.

10. Herzberg B, Campo MJ and Gainor JF: Immune checkpoint inhibitors in non-small cell lung cancer. Oncologist 22: 81-88, 2017.

11. Alsaab HO, Sau S, Alzhrani R, Tatiparti K, Bhise K, Kashaw SK and Iyer AK: PD-1 and PD-L1 checkpoint signaling inhibition for cancer immunotherapy: Mechanism, combinations, and clinical outcome. Front Pharmacol 8: 561, 2017.

12. Brahmer JR, Govindan R, Anders RA, Antonia SJ, Sagorsky S, Davies MJ, Dubinett SM, Ferris A, Gandhi L, Garon EB, et al: The society for immunotherapy of cancer consensus statement on immunotherapy for the treatment of non-small cell lung cancer (NSCLC). J Immunother Cancer 6: 75, 2018. 
13. Arriola E, Wheater M, Galea I, Cross N, Maishman T, Hamid D, Stanton L, Cave J, Geldart T, Mulatero C, et al: Outcome and biomarker analysis from a multicenter phase 2 study of ipilimumab in combination with carboplatin and etoposide as first-line therapy for extensive-stage SCLC. J Thorac Oncol 11: 1511-1521, 2016.

14. Hellmann MD, Callahan MK, Awad MM, Calvo E, Ascierto PA, Atmaca A, Rizvi NA, Hirsch FR, Selvaggi G, Szustakowski JD, et al: Tumor mutational burden and efficacy of nivolumab monotherapy and in combination with ipilimumab in small-cell lung cancer. Cancer Cell 33: 853-861 e854, 2018.

15. Wang RF and Rosenberg SA: Human tumor antigens for cancer vaccine development. Immunol Rev 170: 85-100, 1999.

16. Cohen CJ, Gartner JJ, Horovitz-Fried M, Shamalov K, Trebska-McGowan K, Bliskovsky VV, Parkhurst MR, Ankri C, Prickett TD, Crystal JS, et al: Isolation of neoantigen-specific $\mathrm{T}$ cells from tumor and peripheral lymphocytes. J Clin Invest 125 : 3981-3991, 2015.

17. Sanchez-Paulete AR, Teijeira A, Cueto FJ, Garasa S, Pérez-Gracia JL, Sánchez-Arráez A, Sancho D and Melero I: Antigen cross-presentation and T-cell cross-priming in cancer immunology and immunotherapy. Ann Oncol 28 (Suppl 12): xii44-xii55, 2017.

18. McDonnell AM, Robinson BW and Currie AJ: Tumor antigen cross-presentation and the dendritic cell: Where it all begins? Clin Dev Immunol 2010: 539519, 2010.

19. Gebremeskel S and Johnston B: Concepts and mechanisms underlying chemotherapy induced immunogenic cell death: Impact on clinical studies and considerations for combined therapies. Oncotarget 6: 41600-41619, 2015.

20. Patel RB, Baniel CC, Sriramaneni RN, Bradley K, Markovina S and Morris ZS: Combining brachytherapy and immunotherapy to achieve in situ tumor vaccination: A review of cooperative mechanisms and clinical opportunities. Brachytherapy 17 995-1003, 2018.

21. Botta C, Misso G, Martino EC, Pirtoli L, Cusi MG, Tassone P, Tagliaferri P, Caraglia M and Correale P: The route to solve the interplay between inflammation, angiogenesis and anti-cancer immune response. Cell Death Dis 7: e2299, 2016.

22. Wang D and DuBois RN: Immunosuppression associated with chronic inflammation in the tumor microenvironment. Carcinogenesis 36: 1085-1093, 2015.

23. Kanterman J, Sade-Feldman M and Baniyash M: New insights into chronic inflammation-induced immunosuppression. Semin Cancer Biol 22: 307-318, 2012

24. Wu J and Waxman DJ: Immunogenic chemotherapy: Dose and schedule dependence and combination with immunotherapy. Cancer Lett 419: 210-221, 2018.

25. Shahabi V,Postow MA,Tuck D and Wolchok JD: Immune-priming of the tumor microenvironment by radiotherapy: Rationale for combination with immunotherapy to improve anticancer efficacy. Am J Clin Oncol 38: 90-97, 2015.

26. Apetoh L, Ladoire S, Coukos G and Ghiringhelli F: Combining immunotherapy and anticancer agents: The right path to achieve cancer cure? Ann Oncol 26: 1813-1823, 2015.

27. Nardone V, Pastina P, Giannicola R, Agostino R, Croci S, Tini P, Pirtoli L, Giordano A, Tagliaferri P and Correale P: How to increase the efficacy of immunotherapy in NSCLC and HNSCC: Role of radiation therapy, chemotherapy, and other strategies. Front Immunol 9: 2941, 2018

28. Attia P, Phan GQ, Maker AV, Robinson MR, Quezado MM, Yang JC, Sherry RM, Topalian SL, Kammula US, Royal RE, et al: Autoimmunity correlates with tumor regression in patients with metastatic melanoma treated with anti-cytotoxic T-lymphocyte antigen-4. J Clin Oncol 23: 6043-6053, 2005.

29. Maker AV, Phan GQ, Attia P, Yang JC, Sherry RM, Topalian SL, Kammula US, Royal RE, Haworth LR, Levy C, et al: Tumor regression and autoimmunity in patients treated with cytotoxic T lymphocyte-associated antigen 4 blockade and interleukin 2: A phase I/II study. Ann Surg Oncol 12: 1005-1016, 2005.

30. Correale P, Botta C, Ciliberto D, Pastina P, Ingargiola R, Zappavigna S, Tassone P, Pirtoli L, Caraglia M and Tagliaferri P: Immunotherapy of colorectal cancer: New perspectives after a long path. Immunotherapy 8: 1281-1292, 2016.

31. Correale P, Tagliaferri P, Fioravanti A, Del Vecchio MT, Remondo C, Montagnani F, Rotundo MS, Ginanneschi C, Martellucci I, Francini E, et al: Immunity feedback and clinical outcome in colon cancer patients undergoing chemoimmunotherapy with gemcitabine + FOLFOX followed by subcutaneous granulocyte macrophage colony-stimulating factor and aldesleukin (GOLFIG-1 Trial). Clin Cancer Res 14: 4192-4199, 2008.
32. Kudo-Saito C, Schlom J and Hodge JW: Induction of an antigen cascade by diversified subcutaneous/intratumoral vaccination is associated with antitumor responses. Clin Cancer Res 11: 2416-2426, 2005

33. Hodge JW, Sharp HJ and Gameiro SR: Abscopal regression of antigen disparate tumors by antigen cascade after systemic tumor vaccination in combination with local tumor radiation. Cancer Biother Radiopharm 27: 12-22, 2012.

34. Diem S, Keller F, Ruesch R, Maillard SA, Speiser DE, Dummer R, Siano M, Urner-Bloch U, Goldinger SM and Flatz L: Pembrolizumab-triggered uveitis: An additional surrogate marker for responders in melanoma immunotherapy? J Immunother 39: 379-382, 2016.

35. Wargo JA, Reuben A, Cooper ZA, Oh KS and Sullivan RJ: Immune effects of chemotherapy, radiation, and targeted therapy and opportunities for combination with immunotherapy. Semin Oncol 42: 601-616, 2015.

36. Gibney GT, Weiner LM and Atkins MB: Predictive biomarkers for checkpoint inhibitor-based immunotherapy. Lancet Oncol 17: e542-e551, 2016.

37. Seymour L, Bogaerts J, Perrone A, Ford R, Schwartz LH, Mandrekar S, Lin NU, Litière S, Dancey J, Chen A, et al: iRECIST: Guidelines for response criteria for use in trials testing immunotherapeutics. Lancet Oncol 18: e143-e152, 2017.

38. Correale P, Botta C, Martino EC, Ulivieri C, Battaglia G, Carfagno T, Rossetti MG, Fioravanti A, Guidelli GM, Cheleschi S, et al: Phase Ib study of poly-epitope peptide vaccination to thymidylate synthase (TSPP) and GOLFIG chemo-immunotherapy for treatment of metastatic colorectal cancer patients. Oncoimmunology 5: e1101205, 2015.

39. Martino EC, Misso G, Pastina P, Costantini S, Vanni F, Gandolfo C, Botta C, Capone F, Lombardi A, Pirtoli L, et al: Immune-modulating effects of bevacizumab in metastatic non-small-cell lung cancer patients. Cell Death Discov 2: 16025 , 2016.

40. Correale P, Cerretani D, Remondo C, Martellucci I, Marsili S, La Placa M, Sciandivasci A, Paolelli L, Pascucci A, Rossi M, et al: A novel metronomic chemotherapy regimen of weekly platinum and daily oral etoposide in high-risk non-small cell lung cancer patients. Oncol Rep 16: 133-140, 2006.

41. Pastina P, Nardone V, Croci S, Battaglia G, Vanni F, Bellan C, Barbarino M, Ricci V, Costantini S, Capone F, et al: Anti-cancer activity of dose-fractioned $\mathrm{mPE}+/$ - bevacizumab regimen is paralleled by immune-modulation in advanced squamous NSLC patients. J Thoracic Dis 9: 3123-3131, 2017.

42. Correale P, Botta C, Basile A, Pagliuchi M, Licchetta A, Martellucci I, Bestoso E, Apollinari S, Addeo R, Misso G, et al: Phase II trial of bevacizumab and dose/dense chemotherapy with cisplatin and metronomic daily oral etoposide in advanced non-small-cell-lung cancer patients. Cancer Biol Ther 12: $112-118,2011$.

43. Correale P, Remondo C, Carbone SF, Ricci V, Migali C, Martellucci I, Licchetta A, Addeo R, Volterrani L, Gotti G, et al: Dose/dense metronomic chemotherapy with fractioned cisplatin and oral daily etoposide enhances the anti-angiogenic effects of bevacizumab and has strong antitumor activity in advanced non-small-cell-lung cancer patients. Cancer Biol Ther 9: 685-693, 2010.

44. Pastina P, Nardone V, Botta C, Croci S, Tini P, Battaglia G, Ricci V, Cusi MG, Gandolfo C, Misso G, et al: Radiotherapy prolongs the survival of advanced non-small-cell lung cancer patients undergone to an immune-modulating treatment with dose-fractioned cisplatin and metronomic etoposide and bevacizumab (mPEBev). Oncotarget 8: 75904-75913, 2017.

45. Day D and Hansen AR: Immune-related adverse events associated with immune checkpoint inhibitors. BioDrugs 30: 571-584, 2016.

46. Weber JS, Yang JC, Atkins MB and Disis ML: Toxicities of immunotherapy for the practitioner. J Clin Oncol 33: 2092-2099, 2015.

47. De Remigis A, de Gruijl TD, Uram JN, Tzou SC, Iwama S, Talor MV, Armstrong TD, Santegoets SJ, Slovin SF, Zheng L, et al: Development of thyroglobulin antibodies after GVAX immunotherapy is associated with prolonged survival. Int J Cancer 136: 127-137, 2015.

48. Yuan J, Adamow M, Ginsberg BA, Rasalan TS, Ritter E, Gallardo HF, Xu Y, Pogoriler E, Terzulli SL, Kuk D, et al: Integrated NY-ESO-1 antibody and CD8+ T-cell responses correlate with clinical benefit in advanced melanoma patients treated with ipilimumab. Proc Natl Acad Sci USA 108: 16723-16728, 2011. 
49. Cusi MG, Botta C,Pastina P, Rossetti MG, Dreassi E, Guidelli GM, Fioravanti A, Martino EC, Gandolfo C, Pagliuchi M, et al: Phase I trial of thymidylate synthase poly-epitope peptide (TSPP) vaccine in advanced cancer patients. Cancer Immunol Immunother 64: 1159-1173, 2015.

50. Correale P, Botta C, Rotundo MS, Guglielmo A, Conca R, Licchetta A, Pastina P, Bestoso E, Ciliberto D, Cusi MG, et al: Gemcitabine, oxaliplatin, levofolinate, 5-fluorouracil, granulocyte-macrophage colony-stimulating factor, and interleukin-2 (GOLFIG) versus FOLFOX chemotherapy in metastatic colorectal cancer patients: The GOLFIG-2 multicentric open-label randomized phase III trial. J Immunother 37: 26-35, 2014.

51. Correale P, Botta C, Staropoli N, Nardone V, Pastina P, Ulivieri C, Gandolfo C, Baldari TC, Lazzi S, Ciliberto D, et al: Systemic inflammatory status predict the outcome of k-RAS WT metastatic colorectal cancer patients receiving the thymidylate synthase poly-epitope-peptide anticancer vaccine. Oncotarget 9: 20539-20554, 2018.

52. Guirgis HM: The impact of PD-L1 on survival and value of the immune check point inhibitors in non-small-cell lung cancer; proposal, policies and perspective. J Immunother Cancer 6: 15, 2018.
53. Correale P, Cusi MG, Tsang KY, Del Vecchio MT, Marsili S, Placa ML, Intrivici C, Aquino A, Micheli L, Nencini C, et al: Chemo-immunotherapy of metastatic colorectal carcinoma with gemcitabine plus FOLFOX 4 followed by subcutaneous granulocyte macrophage colony-stimulating factor and interleukin-2 induces strong immunologic and antitumor activity in metastatic colon cancer patients. J Clin Oncol 23: 8950-8958, 2005.

54. Bagley SJ, Kothari S, Aggarwal C, Bauml JM, Alley EW, Evans TL, Kosteva JA, CiunciCA, Gabriel PE, Thompson JC, et al: Pretreatment neutrophil-to-lymphocyte ratio as a marker of outcomes in nivolumab-treated patients with advanced non-small-cell lung cancer. Lung Cancer 106: 1-7, 2017.

55. Banna GL, Passiglia F, Colonese F, Canova S, Menis J, Addeo A, Russo A and Cortinovis DL: Immune-checkpoint inhibitors in non-small cell lung cancer: A tool to improve patients' selection. Crit Rev Oncol Hematol 129: 27-39, 2018.

56. Dudley JC, Lin MT, Le DT and Eshleman JR: Microsatellite instability as a biomarker for PD-1 blockade. Clin Cancer Res 22: 813-820, 2016.

57. Zhu Y, Zhao F, Li Z and Yu J: Current landscape and future directions of biomarkers for predicting responses to immune checkpoint inhibitors. Cancer Manag Res 10: 2475-2488, 2018. 